\title{
PROCESOS DE APRENDIZAJE CAD/CAM: DE ITERACIONES GEOMÉTRICAS A PROTOTIPOS*
}

\author{
CAD/CAM LEARNING PROCESSES: FROM \\ GEOMETRIC ITERATIONS TO PROTOTYPES
}

\author{
FELIPE VÉLIZ FADIC \\ Escuela de Diseño, Universidad Adolfo Ibáñez
}

La aplicación de medios digitales a diferentes actividades y disciplinas — arte, diseño, arquitectura, planificación urbana, entre otrasha evolucionado los procesos creativos y su posterior ejecución aprovechando su capacidad de representación, análisis y desarrollo. Así, la enseñanza de metodologías de trabajo CAD/CAM adquiere relevancia en planes de estudio; capacitando a los estudiantes en el uso de herramientas y su aplicación reflexiva. Este artículo presenta métodos usados en un curso de fabricación digital y expone sus resultados; relacionando etapas de diseño — análogas y digitales-, características de materiales y la producción de objetos y sistemas en un laboratorio de fabricación digital.

CAD/CAM, educación, laboratorios de fabricación, metodologías de enseñanza

Recibido: 3 de junio del 2019

Aprobado: 6 de agosto del 2019

doi: 10.26439/limaq2020.n006.4822

Using digital means in different activities —art, design, architecture, urban planning, among others-and on various scales has prompted the evolution and further execution of creative processes, by taking into advantage its capacity for representation, analysis and development. Thus, teaching CAD/CAM work methodologies has gained importance in university curricula in order to train students to employ tools in a reflective manner. This paper shows the methods used in a digital fabrication course, as well as its outcomes, and relates the stages of analogical and digital design to the materials' characteristics and the production of objects and systems in a digital fabrication laboratory.

CAD/CAM, education, fabrication

laboratories, teaching methodologies

\footnotetext{
*Agradecimientos: a los profesores ayudantes por su colaboración: Nils Reichhard H., Javier López P., Pablo Vintimilla T., Alberto Dentice G. y Lucas Helle P.; a los alumnos del Magíster en Innovación y Diseño de la Universidad Adolfo Ibáñez, generaciones 2015, 2016, 2017 y 2018. Al laboratorio de fabricación de la escuela de diseño y sus encargados, Juan Francisco Luzoro S. y Camilo Rodríguez C., a Nicole Neumann D. y Diego Pinochet P. por sus revisiones y consejos relacionados con los contenidos del artículo.
} 
I INTRODUCCIÓN

La tecnología tiene sus raíces en el pasado. Domina el presente y tiende al futuro. Es un verdadero movimiento histórico; uno de los grandes movimientos que dan forma y representan su época.

Van der Rohe, 1950, p. 6

La manera de concebir un proyecto de arquitectura o diseño ha pasado por diversos cambios. Estos, dirigidos por el constante avance tecnológico aplicado al desarrollo de software, procesos constructivos e investigación de nuevos materiales, complementan cada una de las etapas asociadas al desarrollo de la propuesta con el objetivo de aumentar su eficiencia relacionada a menores costos y tiempos de ejecución, así como también a nuevos métodos de exploración y análisis formal. De esta manera, procesos creativos análogos son apoyados por medios digitales (CAD), coordinación entre especialidades organizada con base en un solo modelo digital (BIM), fabricación de elementos constructivos sistematizados a partir del uso de máquinas de control numérico (CAM), entre otros. Al respecto, Gershenfeld (2012) menciona: "Las raíces de la revolución se remontan a 1952, cuando investigadores del MIT conectaron un antiguo computador a una máquina de fresado... (al usar un computador en reemplazo de procesos manuales se pudieron producir piezas de avión más complejas)” (p. 43).

A principios de los años setenta, el uso de máquinas de control numérico (CNC) relacionado con proyectos de arquitectura, se asocia a la producción de maquetas de estudio o prototipos a escala (Kolarevic, 2001, p. 271). De este modo, evaluar el desempeño frente a variables externas o bien, evitar conflictos de ensamble entre elementos constructivos, son algunas maneras de evidenciar las primeras ventajas en el uso de metodologías de trabajo CAM.

Sin ir más lejos, la oficina de arquitectura de Frank Gehry se vio obligada a complementar su proceso de diseño - cuya principal herramienta es hacer modelos físicos - con software de modelado tridimensional, usados en la industria aeroespacial (Alias / CATIA). Esto, debido a la dificultad de representación bidimensional de la forma, es decir, la planimetría que posteriormente se usará para construir. Así, el desarrollo de la escultura propuesta para los Juegos Olímpicos de Barcelona 1992 — una malla tejida de acero inoxidable de 50 metros de largo - fue asistida, de manera íntegra, por un modelo tridimensional (Shelden, 2002, pp. 26-29).

Desde las primeras incursiones de Gehry en el uso de la metodología de trabajo CAD/CAM hasta nuestros días, tanto oficinas de arquitectura y diseño como profesionales independientes, han complementado sus procesos creativos y de producción bajo esta plataforma. De igual manera, la masificación de estos procesos abandona el ámbito profesional y vincula herramientas digitales a desarrollos personalizados. Por lo tanto, el usuario planifica, diseña, analiza y fabrica. "Del consumidor (el que demanda) al prosumidor (el que 
consume, pero personaliza su producción)" (Herrera y Juárez, 2012, p. 286). De igual forma, Gershenfeld (2012) señala: "Hoy en día, máquinas de control numérico están presentes en casi cualquier producto comercial, ya sea directamente (produciendo todo, desde fundas de computador hasta motores a reacción) o indirectamente (produciendo las máquinas que moldean y estampan productos en masa)" (pp. 43-44). En otros términos, el vínculo entre usuario/profesional/industria y medios digitales de producción/diseño ocurre de manera involuntaria; sin advertir su presencia.

A partir de la democratización y, en consecuencia, el creciente uso de herramientas digitales en procesos creativos y de producción - sumado a los permanentes avances en el desarrollo tecnológico y material- es necesario analizar el rol de estas a nivel formativo, desde su trascendencia en planes de estudio a metodologías usadas.

El presente artículo tiene por objetivo reflexionar acerca de procesos CAD/ CAM aplicados a nivel de educación universitaria en carreras vinculadas al diseño en todas sus escalas - por medio de una descripción detallada (objetivos, métodos y resultados) de un conjunto de ejercicios prácticos realizados en un curso de fabricación digital- y de qué manera estos son adaptados al trabajo práctico en un laboratorio de fabricación, cuya orientación histórica ha sido hacia la ingeniería.

\section{DE NECESIDADES A METODOLOGÍAS}

Desde la aparición de AutoCAD, a finales de 1982, al uso progresivo de la plataforma $\mathrm{BIM}^{1}$, el computador ha sido una herramienta importante en el desarrollo de proyectos. Esto, no solo por aumentar la velocidad de trabajo si no también su capacidad de integrar procesos de diseño - iteraciones geométricas, análisis de elemento finito, elaboración de imágenes- y complementar etapas de producción - modelos tridimensionales para prototipado, optimización de material, simulación de procesos-. En este sentido Achten (2003) señala: "Existe un número importante de oficinas (no solo las más grandes) que usan Computer Aided Architectural Design (CAAD) de manera creativa e innovadora... [usando software de animación y morphing (Lynn, Franken, NOX, Kolatan y MacDonald) o incorporando diversos medios (Gerhy o Eisenman)]" (pp. 74-75). Por consiguiente, nuevas geometrías han derivado de la integración del computador en etapas creativas; siendo estas materializadas a partir del uso de máquinas y post-procesos que, tanto por nivel de acabado como por volumen de producción, tengan la capacidad de desarrollar.

1 A modo de ejemplo: Plambim (Chile), Metodologías y tecnologías para una construcción colaborativa. Programa a 10 años, que tiene como una de sus metas la utilización de la tecnología BIM para el desarrollo y operación de proyectos de edificación e infraestructura pública el año 2020. 
"Se aproxima una nueva revolución, esta vez en fabricación" (Gershenfeld, 2012, p. 43). En la actualidad, la red de laboratorios de fabricación (Fab Lab) está compuesta por aproximadamente 1000, distribuidos en 78 países alrededor de todo el mundo (Fab Foundation, 2019). Estos - cuyo objetivo es ser una plataforma de aprendizaje e innovación - se han posicionado en las universidades con el fin de complementar planes de estudio. De este modo, tanto alumnos como académicos se ven beneficiados al tener la posibilidad de automatizar procesos, construir prototipos que posteriormente serán sometidos a ensayos, el trabajo colaborativo - universidad, industria y comunidad-, entre otros. Sin embargo, la dependencia en el uso de laboratorios de fabricación por parte de alumnos de arquitectura y diseño no es evidente e indiscutible. Esto puede estar justificado por varias razones tales como entender la naturaleza de ambas disciplinas - centradas en sensaciones y percepciones-, su trascendencia frente a otras asignaturas complementarias dentro del plan de estudio (composición, representación, estructura, etcétera) y su aplicación efectiva en el ámbito profesional.

$\mathrm{Al}$ respecto, Feisel y Rosa (2005) analizan el uso de laboratorios en escuelas de ingeniería:

Es útil distinguir entre tres tipos de laboratorios: desarrollo, investigación y educación. Si bien estos tienen varias características en común, hay algunas diferencias fundamentales. Estas, deben ser entendidas para que exista un acuerdo en los objetivos educacionales que se esperan encontrar en los laboratorios de enseñanza... Mientras un laboratorio de desarrollo está dirigido a responder preguntas específicas de importancia inmediata, laboratorios de investigación son usados para buscar ampliar el conocimiento que puede ser generalizado y automatizado, a menudo sin ningún uso específico en mente...Cuando estudiantes [de pregrado] van al laboratorio, no es generalmente para extraer datos necesarios para un diseño, evaluar un nuevo mecanismo o [ampliar nuestro conocimiento acerca del mundo]. (p. 121)

A diferencia de su aplicación en escuelas de ingeniería, el uso de laboratorios de fabricación en escuelas de arquitectura y diseño es reciente. Como describe Celani (2012):

Uno de los primeros laboratorios de fabricación digital en escuelas de arquitectura fue montado por el profesor William Mitchell en el MIT... La primera adquisición del laboratorio fue una impresora 3D, seguido de una cortadora láser...Cortadoras por agua y máquinas de mecanizado CNC estuvieron disponibles a través de un convenio con el departamento de ingeniería mecánica. (p. 473)

Sin embargo, entendiendo contextos y realidades de cada uno, el trabajo colaborativo entre ambos laboratorios ha sido importante para definir necesidades y proyecciones, incluyendo en estas: metodologías y objetivos.

Con relación a la orientación en el uso de laboratorios de fabricación en escuelas de arquitectura, Celani (2012) señala: "El uso explícito de métodos científicos debiera ser incentivado. De hecho, esta es la razón de por qué 
estos son llamados 'laboratorios' ... y no simplemente 'talleres' de fabricación digital" (p. 480). Al respecto, diversos estudios - con base en teorías y demostración de resultados- desarrollan la manera en que el alumno recibe, interpreta y ejecuta la información. Por lo tanto, la metodología usada para la enseñanza de plataformas CAD/CAM y la aproximación de los estudiantes a estas tiene diversas aristas: uso de referentes e interpretación de sus obras (Achten, 2003), categorización con base en el conocimiento previo (Sheppard et al., 2008 como se citó en Celani, 2012), nivel de apoyo por parte de los profesores y lo establecido en los ejercicios planteados (Kirschner, Sweller, Clark, 2006), entre otras.

Asimismo, los objetivos trazados en el aprendizaje no solo deben ser operacionales - uso de máquinas y manejo de software - sino también reflexivos. De esta manera, la aplicación de los conocimientos técnicos adquiridos permite: entender la necesidad en el uso de la metodología CAD/CAM - discriminar cuando requiere ser utilizada-, analizar e interpretar datos obtenidos para mejorar el desempeño del proyecto, integrar a otros medios y herramientas.

\section{CASO DE ESTUDIO}

A continuación, se describe el desarrollo del curso Fabricación Digital Básica (2015-2018) del Magíster en Innovación y Diseño de la Universidad Adolfo Ibáñez. Este introduce a los alumnos - sin conocimiento en procesos de fabricación digital y cuya experiencia académica anterior ha estado asociada a la ingeniería - al uso de máquinas de control numérico y en cómo estas complementan el proceso creativo y de producción.

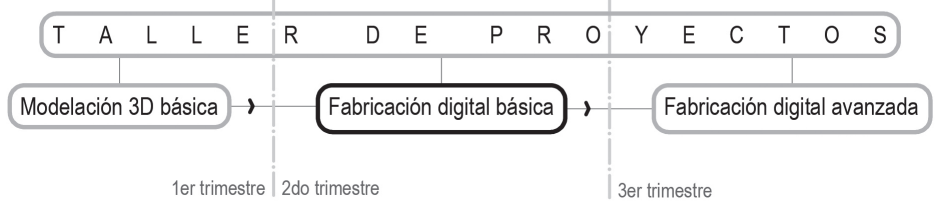

\section{OBJETIVOS}

\section{Figura 1. \\ Distribución de cursos CAD \\ I CAM en el programa de Magíster en Innovación y Diseño}

Fuente: Escuela La metodología aplicada a la asignatura es organizada con el fin de promover el conocimiento técnico (herramientas) y su aplicación reflexiva (métodos). Esta se compone de sesiones prácticas - capacitación de máquinas y/o herramientas digitales-, trabajo en clases - avance de cada proyecto y discusiones con base en estos- y sesiones teóricas -introducción al ejercicio y técnica a desarrollar-. Así, la estructura de cada encargo se apoya en el uso de máquinas específicas del laboratorio de fabricación como medio para producir un objeto tangible que resuelve un problema de diseño. No obstante, el proceso para llegar al elemento final se entiende como un vínculo entre medios análogos y digitales. Por ejemplo, dibujos a mano alzada - posteriormente modelados 
tridimensionalmente - y maquetas de rápida ejecución que permiten anticipar la dificultad de producción en el laboratorio. Más aún, la posproducción de un objeto fabricado por máquinas de control numérico, normalmente, se asocia a procesos manuales, tales como acabado de superficies o complementos en sistemas de ensamble.

Por otra parte, la aproximación material de los alumnos con el objeto terminado, y su fase de producción, permite a estos entender la mutación entre lo digital y físico. En otras palabras, en cómo afectan al resultado final: tolerancias en el modelo tridimensional, desempeño material frente a procesos de fabricación, postprocesos aplicados, entre otros. Al respecto, Pinochet, Labarca y Burdiles (2008) señalan: "La simulación dio a la mente creativa de los arquitectos un nuevo límite de libertad. Sin embargo, esta libertad se mantuvo dentro de los límites de la computadora, ya que esta herramienta... no necesariamente aborda problemas de construcción” (p. 587).

Por lo tanto, los objetivos generales del curso - sumados a los específicos de cada ejercicio- asumen el vínculo inherente entre variables geométricas, materiales y constructivas. Por consiguiente, estos son:

- Promover el trabajo colaborativo entre medios análogos y digitales, entendiendo la necesidad en el uso de cada uno.

- Desarrollar en los estudiantes la capacidad de diseñar en función de cómo se fabrica, considerando ventajas y desventajas en el uso del laboratorio de fabricación.

- Interpretar la condición material como una variable de diseño y aprovechar su característica en procesos de fabricación, asumiendo sus límites y carencias.

\section{DE RUTINAS BIDIMENSIONALES A MATRICES}

\section{Ejercicio 1: Articulaciones - 3 semanas}

Un proyecto, sin importar su escala, se entiende como una suma de partes que lo construyen organizadas a partir de criterios estructurales, redes técnicas, relaciones espaciales, entre otras. Asimismo, estas organizaciones se apoyan en subsistemas; por ejemplo: uniones entre pilares y vigas, instalaciones eléctricas o sanitarias, mobiliarios RTA (ready to assemble). Al respecto, el primer ejercicio a desarrollar presenta a los estudiantes el desafío de diseñar y fabricar ensambles o rótulas que tengan la capacidad de articular dos elementos predefinidos geométricamente y tolerar esfuerzos mecánicos de baja carga. Estos se presentan en formatos regulares (terciado estructural: $300 \mathrm{~mm}$ x $300 \mathrm{~mm}$ x $30 \mathrm{~mm}$ ) e irregulares (pino cepillado seco: $200 \mathrm{~mm} \mathrm{x} 45 \mathrm{~mm} \mathrm{x} 45 \mathrm{~mm}$ ), cuyas caras presentan intervenciones no ortogonales. 
Al finalizar el ejercicio, el alumno debe ser capaz de: (1) diseñar rutinas de mecanizado bidimensional en base a líneas, polilíneas cerradas (área) y puntos identificando aristas, caras y vértices del modelo tridimensional; (2) reconocer variables en el proceso de mecanizado e impresión 3D que inciden en el resultado final y su diferencia, muchas veces imperceptible, con el modelo digital. De esta manera, entender la trascendencia de factores como velocidad de avance y tipo de herramienta, temperaturas, fijación del material en mesa de trabajo, etcétera.

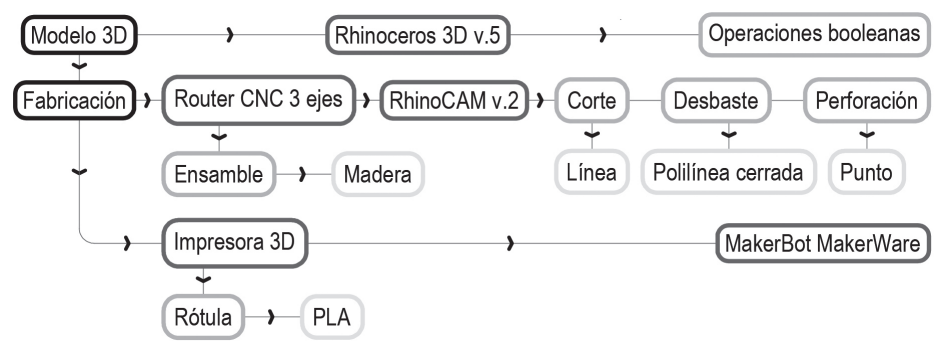

Figura 2.

Organización metodológica CAD/CAM, ejercicio 1

\section{Elaboración} propia

\section{Ejercicio 2: Traslación y estructura - 3 semanas}

El desarrollo de sistemas constructivos ha evolucionado en función de mejorar el desempeño estructural, reducir pérdidas energéticas y aumentar la velocidad de ejecución. Asimismo, la aplicación de medios digitales para el diseño de geometrías continuas e irregulares aumenta la complejidad técnica de estos sistemas. Al respecto, Sánchez (2019) señala: "La base de este movimiento [diseño paramétrico] está vinculada a los avances en fabricación digital y al software de diseño, que desafían la tradición de producción en serie... legado de la revolución industrial" (p. 25).

El segundo ejercicio, define una estrategia de trabajo basada en reglas de crecimiento y una lógica de producción sistematizada. En consecuencia, los alumnos diseñan un solo componente que, a partir de repeticiones, desplazamientos y rotaciones, construye una estructura vertical; además, prescinde de elementos ajenos a la unidad primitiva, es decir, el uso de conectores externos. Así, produce un solo objeto repetidas veces utilizando moldes (madera) y termoplásticos (PET/PAI); por otro lado, analizan el comportamiento mecánico de estos últimos, el cual condiciona la regularidad geométrica y capacidad autoportante de la estructura debido a su característica de expansión o contracción según su formato, tiempos de ejecución, espesores, entre otros.

De esta manera, sumado a los conocimientos adquiridos en el ejercicio anterior, los estudiantes están capacitados para: (3) automatizar procesos de fabricación en serie a partir del uso de moldes y procesos de termoformado; (4) organizar fases de mecanizado tridimensional, diferenciando rutinas de desbaste y acabado; (5) diseñar con base en algoritmos generativos. 
Figura 3

Organización

metodológica

CAD/CAM

ejercicio 2

Elaboración

propia

Figura 4

Organización

metodológica

CAD/CAM,

ejercicio 3

Elaboración

propia

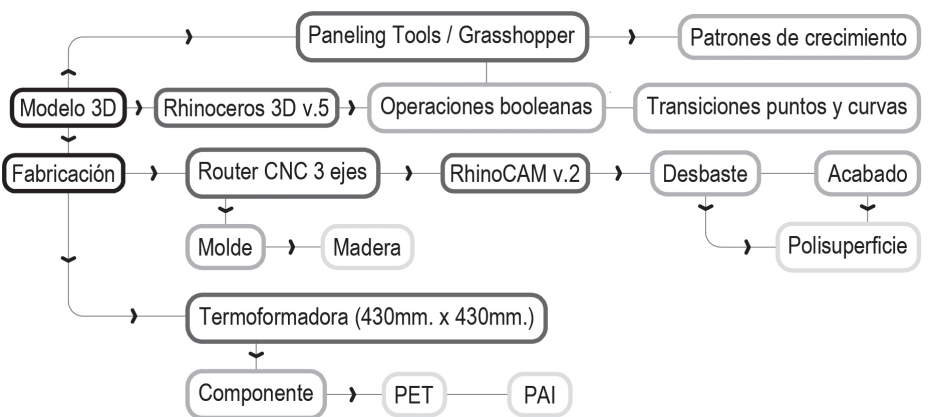

Ejercicio 3: Moldes y vaciado -6 semanas

Mencionada anteriormente, una de las ventajas en el uso del computador en procesos de diseño es su capacidad de iterar sobre una geometría. Esta propiedad, permite simular, analizar y corregir una propuesta bajo una razón estructural, espacial, energética, entre otras; sin embargo, ¿qué sucede si esta cualidad es interpretada desde lo material?, ¿qué condición asume la metodología CAD/CAM en el proceso?, ¿cómo incide esta en el resultado final?

La estructura del ejercicio establece un objeto de diseño predefinido que debe ser modelado tridimensionalmente (pudiendo ser modificado, sin perder su forma primitiva, con el fin de facilitar futuros procesos de producción) luego, obtener tres prototipos en diferentes materialidades; dos de estas predeterminadas (cerámica y resina). Esto, incorpora al proceso protocolos de manipulación dentro del ciclo productivo de manera directa; influyendo en las propiedades mecánicas del objeto resultante. De este modo, el encargo a desarrollar define una serie de estrategias análogas y digitales que organizan el proceso de fabricación a partir de ensayos y análisis; asumiendo el uso de máquinas de control numérico como soporte a procesos manuales. Finalmente, el entendimiento adquirido permite a los estudiantes: (6) diseñar protocolos específicos para materiales a trabajar en estado líquido y su posterior solidificación, (7) desarrollar moldes de vaciado.

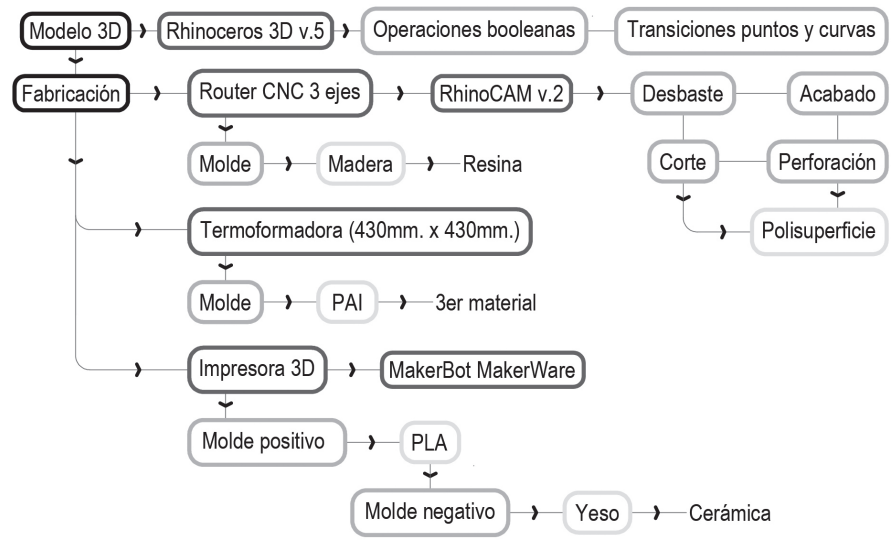




\section{RESULTADOS}

Los ejercicios descritos evidencian una estrategia basada en el hacer; así, los estudiantes adquieren conocimientos de manera práctica, en otras palabras, aplicando de forma experimental una teoría y demostrando sus decisiones a partir de errores y aciertos propios de la experimentación. Al respecto, Yáñez (2016) señala: "Todo aprendizaje siempre constituye un proceso complejo que finalmente se expresa en una modificación de la conducta” (p. 72). A partir de esto, los resultados de cada ejercicio reflejan la capacidad de los estudiantes en adaptar, de manera diferenciada, la información recibida en cada sesión; asimismo, la automatización del trabajo, tanto en el laboratorio de fabricación como fuera de este - modelado tridimensional, elaboración de maquetas, búsqueda de referentes, entre otros- es parcial, con frecuencia condicionada por: carga académica de otras asignaturas, tiempos relacionados a la ejecución de cada ejercicio, incapacidad operativa en el uso de máquinas de control numérico de manera autónoma.
Figura 5.

Prototipos

finales ensamble, ejercicio 1 (2015)

Archivo

fotográfico del autor
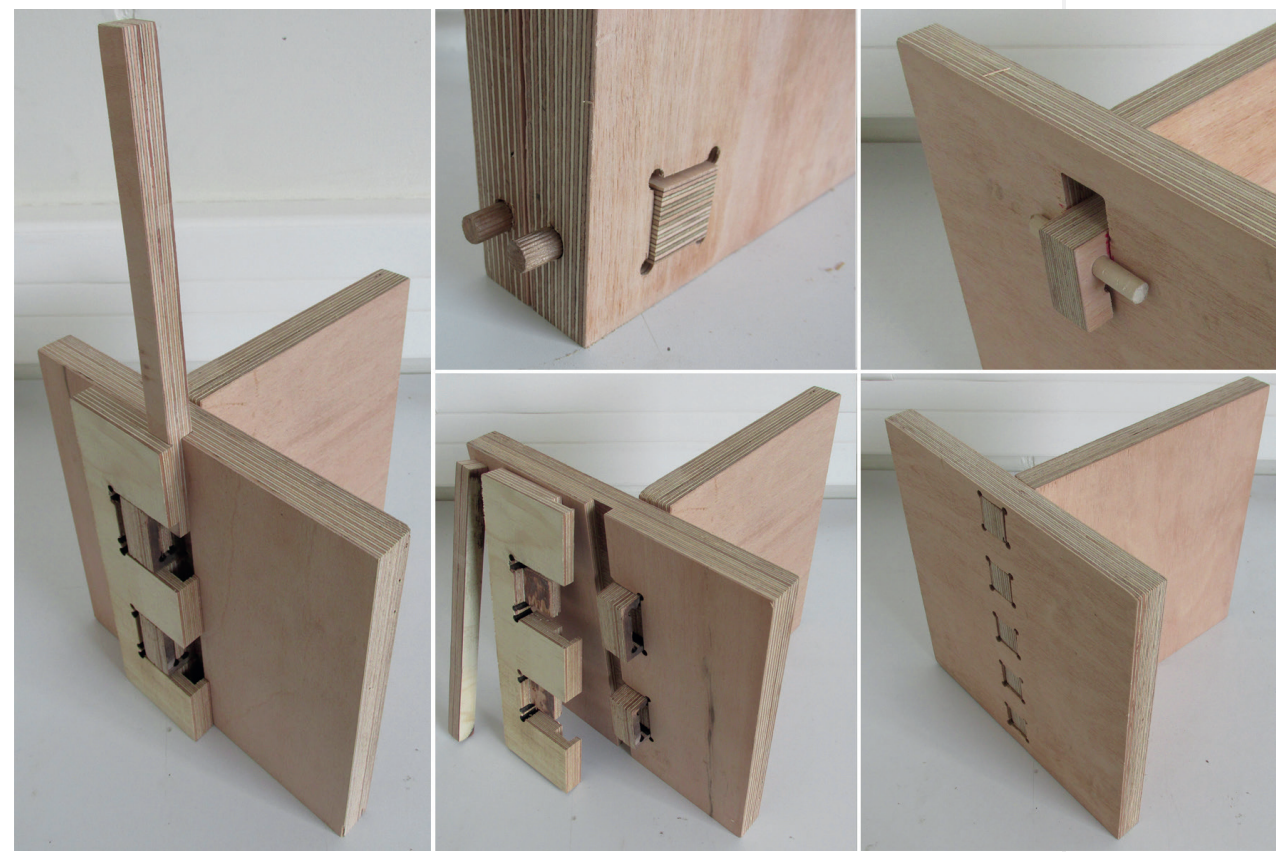

La organización de cada ejercicio establece una distribución gradual de nivel aprendizaje. Así, la evolución del curso asocia temáticas que, en su totalidad, estructuran una aproximación al trabajo en plataformas CAD/CAM; por ejemplo, mecanizado con base en trazados bidimensional y superficies complejas, el dibujo a mano alzada e iteraciones digitales, entendimiento material desde su manipulación, procesos de fabricación sustractiva y aditiva (ya 
sea procedimientos manuales o digitales). Sin embargo, lo descrito anteriormente expone insuficiencias técnicas y metodológicas (propia de estudiantes sin experiencia previa), lo que supone: (1) bajo desarrollo proyectual que permita fundamentar la manera en que se obtiene cierta geometría interpretando variables estructurales, funcionales, estéticas, entre otras, (2) dificultad en representar digitalmente una idea debido al conocimiento limitado de medios para generar un modelo tridimensional. Esto produce modelos insuficientes para procesos CAM —objetos abiertos, intersecciones, polisuperficies discontinuas- y que, en consecuencia, la propuesta original tenga que ser modificada en función de esta limitación.

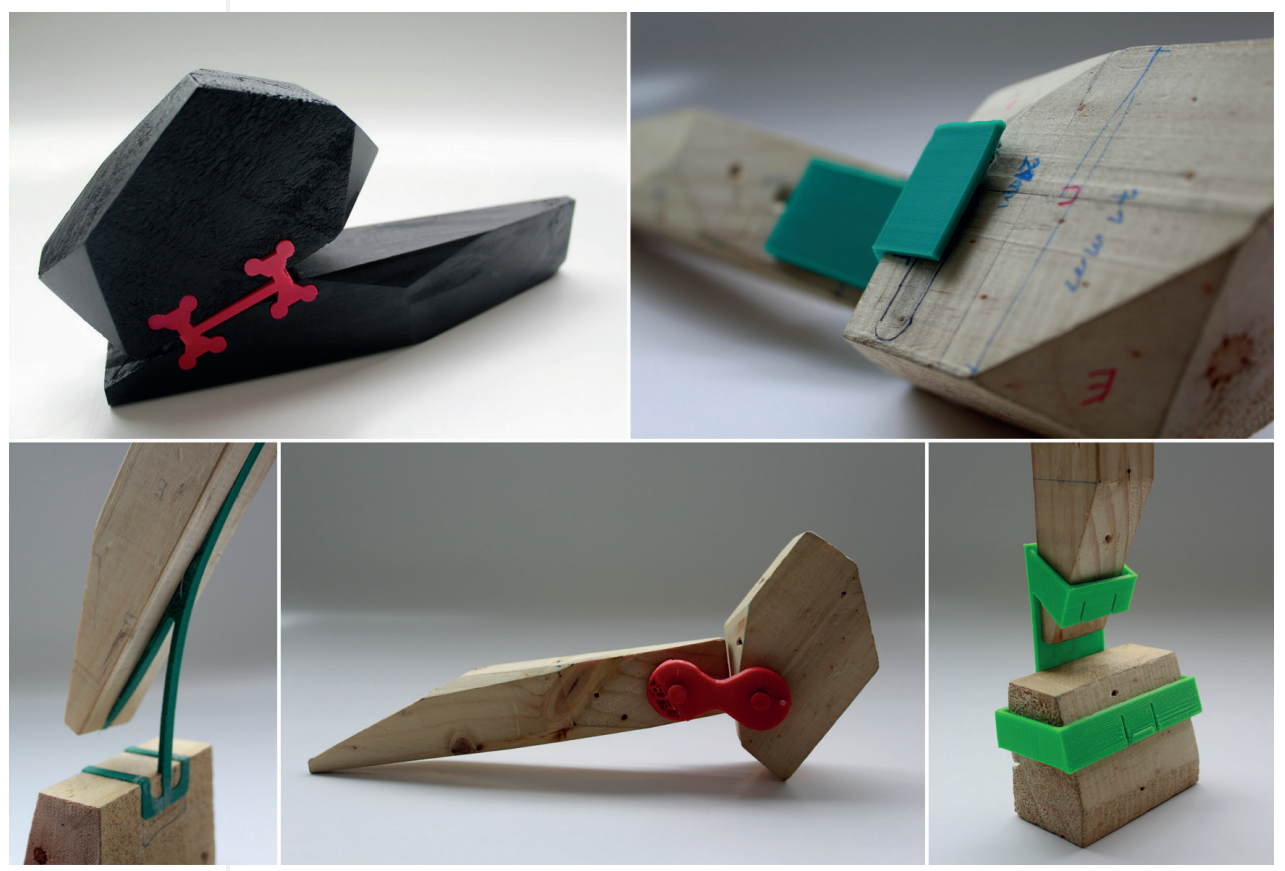

Figura 6. De esta manera, y considerando los objetivos específicos declarados en cada Prototipos finales rótula, ejercicio 1 ejercicio y su alcance al desarrollo del curso, los estudiantes: (1) organizan de mejor manera el trabajo cuando el diseño o geometría a producir es entregado y no debe ser propuesto por ellos. En caso contrario, asignan más tiempo al aspecto del diseño, minimizando el de resolver asuntos relacionados con los procesos de fabricación, (2) desarrollan la capacidad de entender el trabajo con tolerancias propias del material -madera, termoplásticos, termoestables y cerámica - e interpretan aspectos relacionados con el comportamiento mecánico de estos y cómo inciden en el proceso, tanto de modelado digital como fabricación; en contraste, su aplicación tangible resulta irregular, debido a variables geométricas y la cantidad de ensayos realizados con el fin de obtener mejores resultados, (3) relacionan el trabajo entre software de programación y modelo digital, entendiendo aspectos básicos de configuración de 
rutinas de mecanizado; a pesar de esto, no todos están capacitados para operar de manera autónoma las máquinas de control numérico asumiendo que los tiempos de cada estudiante en asimilar la información son diferentes y están determinados por diversos factores, no solo técnicos. Respecto a esto, Yáñez (2016) describe cada una de las fases que deben relacionarse entre sí para alcanzar niveles óptimos de aprendizaje; entre estas están: motivación, interés, comprensión, transferencia, etcétera.
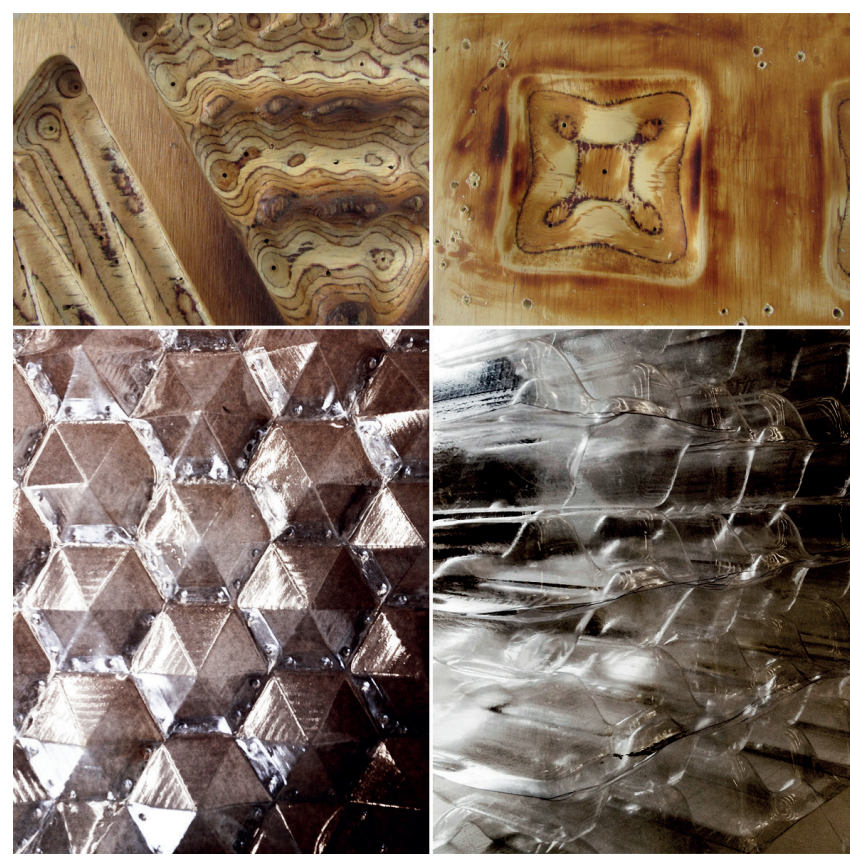

\section{CONCLUSIONES}

La enseñanza de procesos CAD/CAM en escuelas de arquitectura o diseño responde a la necesidad de los estudiantes de entender y aplicar — a diferentes escalas y de manera reflexiva- los constantes cambios que experimenta el desarrollo profesional en términos de estrategias proyectuales y constructivas. Así, la metodología basada en el uso constante de máquinas y software forma hábitos que, de manera involuntaria, los alumnos adaptan y utilizan para resolver distintas variables que forman parte de un proyecto. De igual forma, el uso de laboratorios de fabricación enfocado a complementar procesos de producción y su vínculo con métodos análogos o procedimientos artesanales favorece el acercamiento, por parte de los estudiantes, a máquinas de control numérico, procesos de automatización, etcétera; evidenciando una aplicación real por sobre otras que requieren un conocimiento que, todavía, ellos no tienen. Por consiguiente, y dependiendo de la experiencia o nivel de conocimiento previo de los estudiantes, los objetivos deben ser establecidos y
| Figura 7.

Moldes y prototipos finales, ejercicio 2 (2015)

Archivo fotográfico del autor 
diferenciados por sobre todo lo demás, a partir de aspectos vinculados con su aplicación y no en términos operativos; entendiendo que el uso de máquinas de control numérico y software de diseño digital —más allá de diferencias en interfaces de programación, modelos, tamaños máximos de trabajo, resolución, entre otras - es un medio para desarrollar una propuesta.
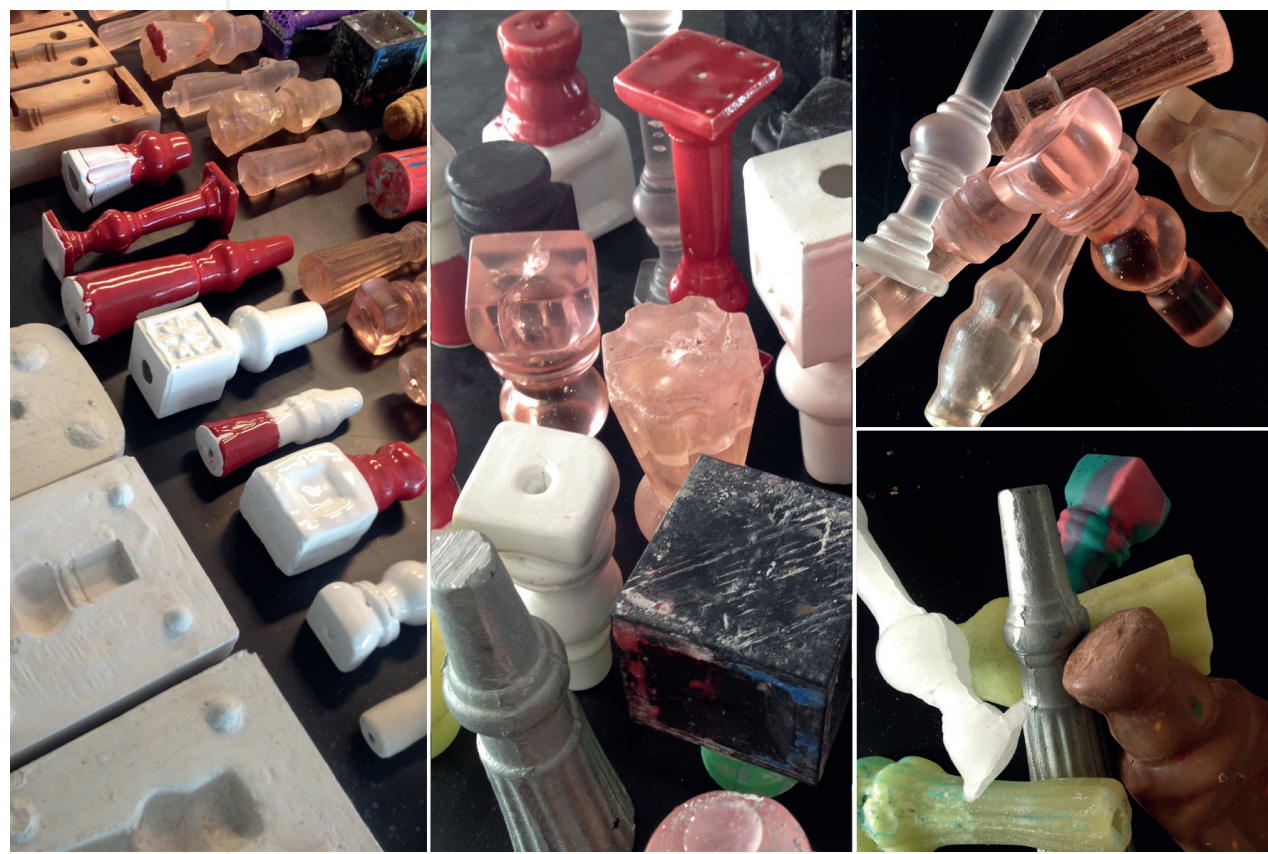

Figura 8.

Moldes y pro-

totipos finales, ejercicio 3 (2017)

Fuente: archivo personal

\section{REFERENCIAS}

Achten, H. (2003). New Design Methods for Computer Aided Architectural Design Methodology Teaching. International Journal of Architectural Computing (IJAC), 1(1)

Celani, G. (2012). Digital Fabrication Laboratories: Pedagogy and Impacts on Architectural Education. Nexus Network Journal, Architecture and Mathematics, 14(3).

Feisel, L., Rosa, A. (2005). The Role of the Laboratory in Undergraduate Engineering Education. Journal of Engineering Education (JEE), 94(1).

Gershendfeld, N. (2012). How to Make Almost Anything: The Digital Fabrication Revolution. Foreign Affairs. Estados Unidos.

Herrera, P., Juárez, B. (2012). Perspectivas en los laboratorios de fabricación digital en Latinoamérica. SIGRADI. Fortaleza, Brasil. 
Kirschner, P., Sweller, J. y Clark, R. (2006). Why Minimal Guidance During Instruction Does Not Work: An Analysis of the Failure of Constructivist Discovery, Problem-Based, Experiential, and Inquiry-Based Teaching. Educational Psychologist, 4(2).

Kolarevic, B. (2001). Digital Fabrication: Manufacturing Architecture in the Information Age. ACADIA. Nueva York.

Pinochet, D., Labarca, C. y Burdiles, M. (2008). Dichotomy Between Digital Project and Real Project Execution. eCAADe. Antwerpen.

Puente. M.(ed) (2006). Conversaciones con Mies van der Rohe. Arquitectura y Tecnología, (6-7). Barcelona: Gustavo Gili.

Sánchez, J. (2019). Architecture for the Commons: Participatory Systems in the Age of Platforms. Architectural Design (AD), 89(2).

Shelden, D. (2002). Digital Surface Representation and the Constructibility of Gehry's Architecture. Boston: Massachusetts Institute of Technology.

Yáñez, P. (2016). El Proceso de aprendizaje: fases y elementos fundamentales. Revista San Gregorio, 11(1). 
